\title{
Post-operative opioid consumption after orthopedic surgery using Sublingual Patient Controlled Analgesia: effects of different anesthetic techniques
}

Coluzzi $F^{1}$, Bramato $A^{1}$, Scerpa $M^{1}$, Corona $A^{1}$, Favetti $F^{1}$, De Martino $E^{1}$, Celidonio $L^{1}$, Locarini $P^{1}$ ${ }^{1}$ Unit of Anesthesia, Intensive Care and Pain Therapy Dept. Medical and Surgical Sciences and Biotechnologies SAPIENZA University of Rome, Polo Pontino - Latina, Italy

\section{Background:}

Hip and knee osteoarthrosis are among the most common musculoskeletal disease in adults. An estimated 7.2 million Americans have undergone total hip arthroplasty (THA) and total knee arthroplasty (TKA). Acute postoperative pain after hip and knee replacement surgeries has been reported as moderate-tosevere by most of patients [1]. Different analgesic protocols have been proposed, including systemic analgesia and regional analgesic techniques. Sufentanil Sublingual Tablet System Zalviso ${ }^{\circledR}$ (SSTS) is an innovative non-invasive patient controlled analgesia (PCA) that has been recently shown to be a safe and effective alternative for the management of severe pain after orthopedic surgery [2]. Aim of this study is to evaluate the effects of different anesthetic techniques on post-operative analgesic opioid consumption in patients who underwent THA and TKA and received SSTS for post-operative analgesia.

\section{Methods:}

Adults aged $\geq 18$ years, who had undergone THA and TKA, received SSTS $15 \mathrm{mcg}$, with 20-minute lockout interval, for the first 72 hours after surgery. In retrospective analysis, patients were grouped according to the kind of surgery and the type of anesthesia. THA was performed under spinal anesthesia (SA) using hyperbaric bupivacaine $0.5 \%$, or general balanced anesthesia (GA) using inhaled sevoflurane and continuous infusion of remifentanil. TKA was conducted under SA, using hyperbaric bupivacaine $0.5 \%$, or single-shot peripheral nerve block (PNB - sciatic and femoral nerve blocks) using Ropivacaine $0.75 \%$.
Data were collected at baseline (T0) and from day 1-3 after surgery $(\mathrm{T} 1, \mathrm{~T} 2, \mathrm{~T} 3)$. Number of total daily doses and timing for the first dose were recorded.

\section{Results:}

28 patients were included after THA $(n=17 ; 60.7 \%)$ and TKA $(n=11 ; 39.3 \%) .3$ patients prematurely interrupted treatment and were excluded in the final analysis. Patients undergoing TKA reported a higher post-operative opioid consumption, compared with those undergoing THA (31.3 vs 23.9 doses in 72 hours). In THA, SA and GA were associated with a similar postoperative opioid consumption, 25.3 vs 22.5 doses respectively. Similarly, in TKA, no differences were observed in total opioid consumption in patients undergoing PNBs and SA, which resulted in 32.5 vs 30 doses, respectively (Fig. 1). Timing for the first SSTS dose was similar in both groups in patients undergoing SA (75.9 vs $74 \mathrm{~min}$, in THA and TKA respectively). Conversely, mean timing for the first dose was significantly shortened by GA in THA (27.5 min) and delayed in PNB in TKA (216.7 min) (Fig.2). In both groups (THA and TKA), patient global assessment (PGA) was "excellent" or "very good" in more than $80 \%$ of patients.

\section{Conclusions:}

Total opioid consumption after major orthopedic surgery was not affected by regional anesthesia. Total daily opioid dose was more strictly related to the kind of orthopedic surgery (TKA > THA), rather than to the type of anesthesia. SSTS was shown to be an effective alternative for post-operative pain management, reducing risk of residual blocks and fastening patient recovery.
Fig1: Opioid consumption (SSTS doses) after THA and TKA

Total SSTS Doses in THA

Total SSTS Doses in TKA

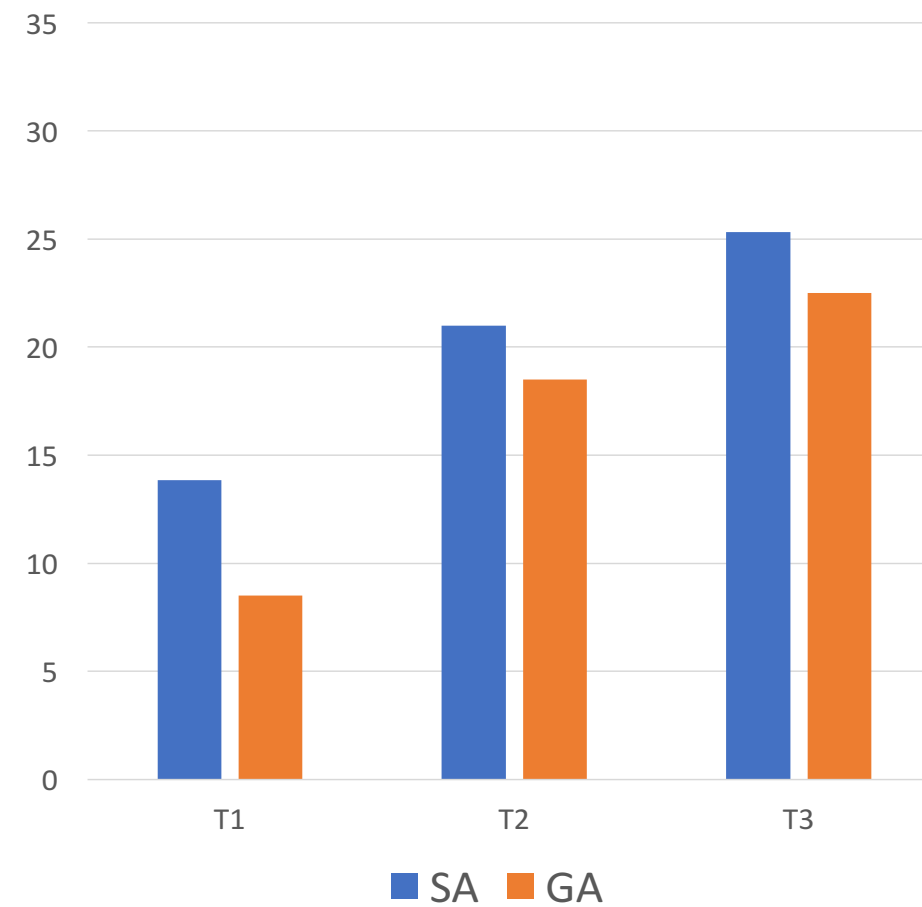

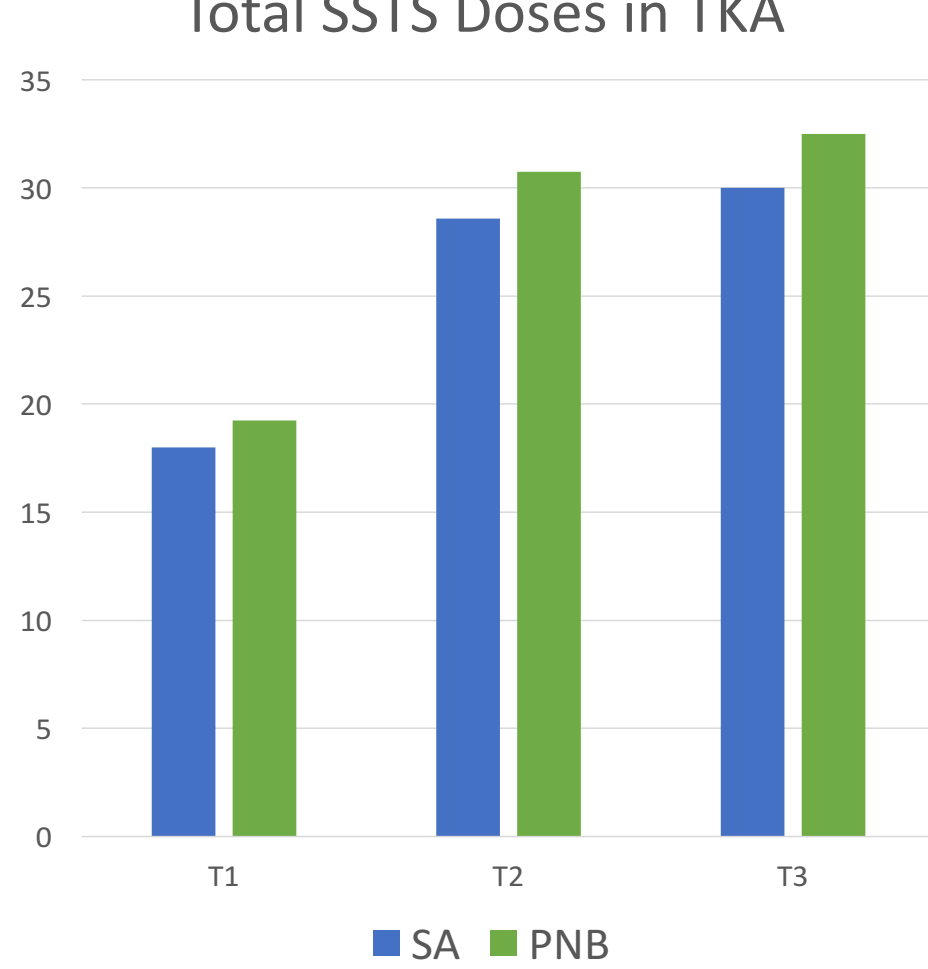

Fig 2: Timing for the first post-operative dose

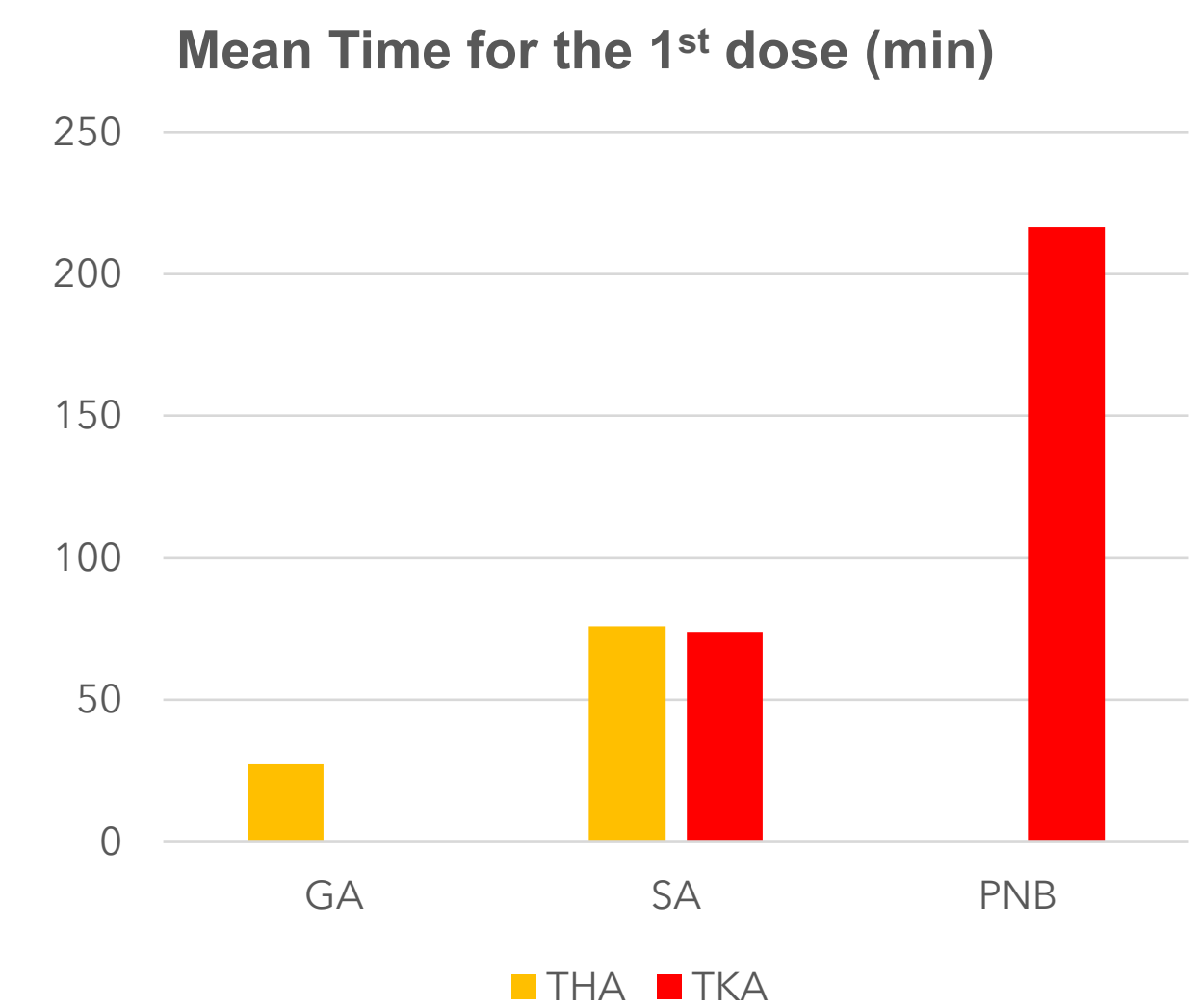

